\title{
Nonlinear Magnetohydrodynamics from Gravity
}

\author{
James Hansen五 and Per Kraus 2 \\ Department of Physics and Astronomy, UCLA, \\ Los Angeles, CA 90095-1547, USA.
}

\begin{abstract}
We apply the recently established connection between nonlinear fluid dynamics and AdS gravity to the case of the dyonic black brane in $\mathrm{AdS}_{4}$. This yields the equations of fluid dynamics for a $2+1$ dimensional charged fluid in a background magnetic field. We construct the gravity solution to second order in the derivative expansion. From this we find the fluid dynamical stress tensor and charge current to second and third order in derivatives respectively, along with values for the associated transport coefficients.
\end{abstract}

November, 2008

\footnotetext{
1 jhansen@physics.ucla.edu

2 pkraus@ucla.edu
} 


\section{Introduction}

Work over the past year has established an elegant connection between long wavelength disturbances of asymptotically AdS black brane solutions and relativistic fluid dynamics. The relativistic Navier-Stokes equations emerge from Einstein's equations applied to metrics with slowly varying thermodynamic parameters [1.22]. In the context of the AdS/CFT correspondence, this construction also provides an efficient means of computing transport coefficients in the dual CFT. The details of this connection have been worked out for pure gravity in arbitrary dimensions [1, 3, [. 4 .5], Einstein-Maxwell theory in $4+1$ dimensions [6, 7, 8], and gravity coupled to a scalar field in $4+1$ dimensions [9]. There is also an extensive earlier literature on the study of transport coefficients within the AdS/CFT correspondence; see [10] for a review and further references.

In this paper we consider dyonic black brane solutions in asymptotically $\mathrm{AdS}_{4}$ spacetimes. These are solutions of Einstein-Maxwell theory in four spacetime dimensions carrying nonzero charge densities and boundary magnetic fields. The computation of transport coefficients for the dyonic black brane has already received significant attention in light of its connection to quantum critical points in $2+1$ dimensional condensed matter systems [11, 12, 13, 14, 15]. The approach taken in these works differs from ours, and yields results with a different (but overlapping) regime of validity, as we discuss further below.

Corresponding to the bulk metric and gauge field are a boundary stress tensor and current, which obey the conservation laws 3

$$
\nabla_{\mu} T^{0 \mu}=0, \quad \nabla_{\mu} T^{i \mu}=B \epsilon^{i j} J_{j}, \quad \nabla_{\mu} J^{\mu}=0,
$$

where $B$ represents the constant magnetic field on the boundary. The stress tensor is also traceless due to scale invariance. Fluid dynamics is a description of the long wavelength evolution of conserved charge densities. At vanishing magnetic field, the conserved charges are energy, momentum, and electric charge. Also, by "long wavelength" one means long compared to the mean free path, which is in turn proportional to the inverse temperature, $l_{T} \sim \frac{1}{T}$. One then sets up an expansion in terms of the small parameter $l_{T} \partial_{\mu}$, where the derivative acts on the conserved charge densities. At nonzero magnetic field the story changes. First, there is now a second length scale in the problem, $l_{B} \sim \frac{1}{\sqrt{B}}$. Second, we see from (1.1) that the momentum current $J^{\left(p_{i}\right) \mu}=T^{i \mu}$ is no longer conserved. At small $B$ there is a separation of the two length scales, $l_{T} \ll l_{B}$, and the appropriate fluid dynamical variables depend on the length scale being probed. At intermediate length scales $l_{T} \ll l \ll l_{B}$, we should treat $l_{T} \partial_{\mu}$ as a small parameter but work to all orders in $l_{B} \partial_{\mu}$. Also, in this regime momentum is approximately conserved, and so the momentum density should be kept as a hydroynamical variable; this is the approach used in 11, 12, 13, 14, 15]. At

3 There is also the dual current, $\tilde{J}^{\mu}=\epsilon^{\mu \alpha \beta} \nabla_{\alpha} J_{\beta}$, but this will play no role in our discussion. 
the largest length scales, $l \gg l_{B}$, momentum is no longer an independent hydrodynamical variable, but is instead fixed in terms of the energy and electric charge densities. Thus, in this regime we can formulate a simpler effective theory by "integrating out" the momentum variables. This is the approach we take in this paper. Note that for large magnetic fields, such that $l_{B} \sim l_{T}$, there is no separation of scales and it is clearly inappropriate to include the momenta, since they are non-conserved at leading order. Keeping the momentum variables in this case is analogous to retaining a very massive particle in a low energy effective field theory; it is not incorrect to do so, but it is inefficient. What is being said here is not that the momentum densities are being set to zero (they are not), but rather that their values are fixed in terms of the charge and energy densities, and so are not independent.

Our goal is therefore to solve the Einstein-Maxwell equations iteratively in an expansion with respect to derivatives of the energy and electric charge densities, treating both $l_{B} \partial_{\mu}$ and $l_{T} \partial_{\mu}$ as small expansion parameters. We carry out this task to second order in the derivative expansion, following the methods applied to previous examples. This gives us the stress tensor to second order. Using the stress tensor conservation equation, we can then read off the current to third order in derivatives. This gives us results for a host of new transport coefficients beyond what was computed in [11,12,13, 14, 15, including nonlinear terms. On the other hand, for the reasons described above, we are restricted to the small frequency/wavelength regime, in contrast to [11,12,13, 14, 15 where full AC results can be found at the level of linear response (at least numerically). Thus, the two approaches are complementary.

Magnetohydrodynamics is of course a highly developed subject, with many important applications to astrophysics and plasma physics. Before trying to compare with this literature it is important to bear in mind a few points. First, here we are considering the fluid dynamics of a conformal field theory, which is quite different than the gas of charged particles considered in most applications. Second, most discussion of magnetohydrodynamics are in the context of a dynamical electromagnetic field coupled to the charged fluid, whereas here we consider a nondynamical external magnetic field. Note, however, that that this should be a good model of the dynamical case if the strength of the magnetic field is taken to be very large while holding the charge fixed, since then we should be able to neglect backreaction from the fluid.

It should be noted that although we usually discuss the case in which only a magnetic field is present and not an electric field, this is not really a restriction since we can always apply a Lorentz boost to our solutions to obtain fluid flows with any electric and magnetic fields obeying $E^{2}<B^{2}$. 


\section{Structure of fluid dynamics}

We begin with a general discussion of the structure of our fluid dynamics, independent of the relation to AdS gravity. We consider a general $2+1$ dimensional conformal field theory with a conserved current $J_{\mu}$ coupled to a constant external magnetic field $B$. We denote the coordinates of our three dimensional manifold $x^{\mu}$.

\subsection{Derivative expansion}

There are two conserved quantities in our theory, charge and energy. In local thermal equilibrium the corresponding charge and energy densities of our fluid, $\rho$ and $\epsilon$, are slowly varying functions of the coordinates, $x^{\mu}$. The current and stress tensor admit an expansion in derivatives of $\epsilon$ and $\rho$, and the equations of fluid dynamics are then given by (11.1). All that remains to specify the theory are the transport coefficients of the various terms in the derivative expansions of $T^{\mu \nu}$ and $J^{\nu}$.

By definition, we have

$$
T^{00}=\epsilon, \quad J^{0}=\rho,
$$

with no derivative corrections. ${ }^{-1}$ To proceed, we need to work out the derivative expansion of the remaining components, $T^{0 i}, T^{i j}$, and $J^{i}$.

Our task is simplified by noting that the equations $\partial_{\mu} T^{i \mu}=B \epsilon^{i j} J_{j}$ imply that the spatial components of the current at $n$th order are related to the stress tensor at order $n-1$,

$$
J_{i}^{(n)}=-\frac{1}{B} \epsilon_{i j}\left(\partial_{0} T^{(n-1) j 0}+\partial_{k} T^{(n-1) j k}\right) .
$$

With this in mind, the remaining equations of fluid dynamics reduce to

$$
\begin{aligned}
& \partial_{0} \rho+\partial_{i} J^{i}=0, \\
& \partial_{0} \epsilon+\partial_{i} T^{0 i}=0 .
\end{aligned}
$$

At zeroth order, tracelessness and rotational symmetry imply

$$
T^{(0) 0 i}=0, \quad T^{(0) i j}=\frac{1}{2} \epsilon \delta^{i j}, \quad J^{(0) i}=0 .
$$

At first order we have

$$
\begin{aligned}
& T^{(1) 0 i}=\left(a_{1} \delta_{i j}+a_{2} \epsilon_{i j}\right) \partial_{j} \epsilon+\left(a_{3} \delta_{i j}+a_{4} \epsilon_{i j}\right) \partial_{j} \rho, \\
& T^{(1) i j}=0 \\
& J^{(1) i}=-\frac{1}{2 B} \epsilon_{i j} \partial_{j} \epsilon,
\end{aligned}
$$

4 In discussions of fluid dynamics in which the fluid velocity appears as a hydrodynamical variable the charge and energy densities are usually defined in the fluid rest frame. In our case there is a preferred frame, defined such that there is a magnetic field but no electric field, and our densities are always measured in this frame. 
where we used (2.2). The coefficients $a_{1,2,3,4}$ are functions of $\epsilon$ and $\rho$ but not their derivatives.

We next consider terms of second order in derivatives. Here it is convenient to combine the two quantities $\epsilon$ and $\rho$ into a two component object

$$
q_{a}=(\epsilon, \rho) .
$$

The second order spatial current $J^{(2) i}$ is gotten by applying (2.2) to (2.5). Rotational invariance implies that $T^{(2) 0 i}$ can be built out of the following structures:

$$
\partial_{0} \partial_{i} q_{a}, \quad \partial_{0} q_{a} \partial_{i} q_{b}, \quad \epsilon_{i j} \partial_{0} \partial_{j} q_{a}, \quad \partial_{0} q_{a} \epsilon_{i j} \partial_{j} q_{b}
$$

Now, in general, if one is working up to $n$th order, it is permissible to use the zeroth order equations of motion to simplify the current and stress tensor at $n$th order, since the error will be of at least order $n+1$. The zeroth order equations of motion yield $\partial_{0} \epsilon=\partial_{0} \rho=0$. Noting that every term in (2.7) involves at least one time derivative acting on either $\epsilon$ or $\rho$, we see that we can set $T^{(2) 0 i}=0$. This leaves $T^{(2) i j}$, which has the structure

$$
T^{(2) i j}=b_{1}^{a}\left[\partial_{i} \partial_{j} q_{a}\right]^{s t}+b_{2}^{a}\left[\epsilon_{i k} \partial_{k} \partial_{j} q_{a}\right]^{s t}+b_{3}^{a b}\left[\partial_{i} q_{a} \partial_{j} q_{b}\right]^{s t}+b_{4}^{a b}\left[\epsilon_{i k} \partial_{k} q_{a} \partial_{j} q_{b}\right]^{s t}
$$

where we are using the following notation for the symmetric traceless part of a spatial tensor:

$$
\left[M_{i j}\right]^{s t}=\frac{1}{2}\left(M_{i j}+M_{j i}-\delta_{i j} M_{k k}\right) .
$$

Finally, the third order current is obtained by applying (2.2) to the second order stress tensor.

Our fluid dynamics has thus been specified in terms of the coefficients $a_{1,2,3,4}$ and $b_{1,2,3,4}$, which are themselves functions of $\epsilon$ and $\rho$. In the rest of this paper we will see that the boundary stress tensor and current obtained from the Einstein-Maxwell equations in $4+1$ dimensions falls into the above framework and yields specific formulas for the coefficients. In particular, $a_{1,2,3,4}$ are given in equation (6.14); $b_{1,2}$ are given in equation (6.29); and $b_{3,4}$ are given in equations (6.34) and (6.35).

In the above we considered fluid dynamics in a magnetic field but vanishing electric field. But by applying a Lorentz boost we can turn on an electric field, subject to the condition $\vec{E}^{2}<B^{2}$. As a simple example, consider an infinitesimal Lorentz boost with velocity $v^{i}:\left(t^{\prime}=t+v \cdot x, x^{i}=x^{i}+v^{i} t\right)$. The magnetic field is unchanged, and the new electric field is $E_{i}=-B \epsilon_{i j} v^{j}$. To keep things simple, let's also work to lowest order in both $v^{i}$ and the derivative expansion (i.e. consider $v^{i} \sim \partial_{i} \epsilon \sim \partial_{i} \rho$ ), in which case we can write the current after the boost as

$$
J_{i}=-\frac{1}{2 B} \epsilon_{i j} \partial_{j} \epsilon-\frac{\rho}{B} \epsilon_{i j} E_{j}
$$


which displays the Nernst effect as well as the Hall conductivity. We can also write down an expression for the energy flux at this order,

$$
T^{(1) 0 i}=\left(a_{1} \delta_{i j}+a_{2} \epsilon_{i j}\right) \partial_{j} \epsilon+\left(a_{3} \delta_{i j}+a_{4} \epsilon_{i j}\right) \partial_{j} \rho+\frac{3 \epsilon}{B} \epsilon_{i j} E_{j}
$$

By performing a finite boost and keeping terms of higher order in the derivative expansion, one can of course easily read off the generalized version of these results, if desired.

We have chosen to formulate our fluid dynamics in terms of the energy and charge density, since these have a direct physical significance and appear naturally in the gravitational description. But we can alternatively work in terms of the temperature and chemical potential. The definition of these is subject to some ambiguity in a non-equilibrium context. One simple choice is to use the same definition between the energy/charge densities and the temperature/potential as appears in the equilibrium case, without any derivative corrections. The relation between these sets of variables of course depends on the theory in question. For the theory corresponding to the dyonic black brane the relations will be given in the following section.

\subsection{Normal modes}

We now look for normal mode solutions by solving the linearized equations of motion following from (2.3). Dropping nonlinear terms, these become

$$
\begin{aligned}
& \partial_{0} \rho+\frac{a_{2}}{B} \vec{\nabla}^{2} \partial_{0} \epsilon+\frac{a_{4}}{B} \vec{\nabla}^{2} \partial_{0} \rho+\frac{b_{2}^{\epsilon}}{2 B}\left(\vec{\nabla}^{2}\right)^{2} \epsilon+\frac{b_{2}^{\rho}}{2 B}\left(\vec{\nabla}^{2}\right)^{2} \rho=0 \\
& \partial_{0} \epsilon+a_{1} \vec{\nabla}^{2} \epsilon+a_{3} \vec{\nabla}^{2} \rho=0 .
\end{aligned}
$$

Inserting plane wave fluctuations on top of the constant background, $\epsilon+\delta \epsilon e^{i \vec{k} \cdot \vec{x}-i \omega t}$ and $\rho+\delta \rho e^{i \vec{k} \cdot \vec{x}-i \omega t}$, we find

$$
\begin{aligned}
& \left(-i \omega a_{2}-\frac{b_{2}^{\epsilon}}{2} k^{2}\right) k^{2} \delta \epsilon+\left(i B \omega-i a_{4} \omega k^{2}-\frac{b_{2}^{\rho}}{2} k^{4}\right) \delta \rho=0 \\
& \left(i \omega+a_{1} k^{2}\right) \delta \epsilon+a_{3} k^{2} \delta \rho=0 .
\end{aligned}
$$

Setting the determinant to zero we find two modes

$$
\begin{aligned}
\omega & \approx i a_{1} k^{2}+\cdots, \quad \text { with } \delta \rho \approx 0 \\
\omega & \approx-\frac{i}{2} \frac{\left(a_{1} b_{2}^{\rho}+a_{3} b_{2}^{\epsilon}\right)}{a_{1} B} k^{4}+\cdots, \quad \text { with } a_{1} \delta \epsilon+a_{3} \delta \rho \approx 0 .
\end{aligned}
$$

In our gravitational computation we will find $a_{1}<0$ so that the first mode is strictly decaying in time. But for the second mode we find that $\frac{\left(a_{1} b_{2}^{\rho}+a_{3} b_{2}^{\epsilon}\right)}{a_{1} B}$ has the same sign as $3\left(B^{2}-\rho^{2}\right) a^{4}+\left(B^{2}+\rho^{2}\right)\left(3 B^{2}-\rho^{2}\right)$. Since this can have either sign, the $k^{4}$ mode can be either decaying or growing in time, depending on the values of $\rho$ and $\epsilon$. 


\subsection{Comparison with other approaches}

In this section we have developed a formalism for studying magnetohydrodynamics which, on its surface at least, represents a significant departure from existing treatments in the literature. While our definitions of the transport coefficients are based on following consistently the logic of hydrodynamic expansions, we would be remiss not to discuss in detail the relation between the different approaches. The purpose of this section is to provide that discussion, together with a dictionary between the formalisms where appropriate. We conclude this section with a careful treatment of the shear viscosity and its universal relationship with the entropy density.

In most discussions of relativistic conformal hydrodynamics the starting point is a Lorentz covariant parametrization of the stress tensor, 5 organized in a derivative expansion with respect to the fluid velocity $u^{\mu}$ as follows:

$$
\begin{aligned}
& T^{(0) \mu \nu}=\epsilon u^{\mu} u^{\nu}+P \Delta^{\mu \nu} \\
& T^{(1) \mu \nu}=\eta\left(\Delta^{\mu \alpha} \Delta^{\nu \beta}\left(\partial_{\alpha} u_{\beta}+\partial_{\beta} u_{\alpha}\right)-\Delta^{\mu \nu} \partial_{\alpha} u^{\alpha}\right),
\end{aligned}
$$

with

$$
\Delta^{\mu \nu}=\eta^{\mu \nu}+u^{\mu} u^{\nu}
$$

Here, we have listed all of the transport coefficients present to first order in derivatives of the velocity. A particularly good discussion of the relationship between these expressions and the dynamics of charged fluids can be found in [6]. We note that the charge density does not enter explicitly until the next order of the derivative expansion.

The form (2.15) can be justified for fluids in Lorentz invariant backgrounds. In particular, in thermal equilibrium the fluid can be taken to have any constant velocity, and the hydrodynamic expansion then promotes these constants to slowly varying functions. The assumption of a Lorentz invariant background clearly does not hold in the presence of an external magnetic field; nevertheless, (2.15) is taken as the starting point in other treatments of conformal linear magnetohydrodynamics from AdS/CFT [13,15, 16]. As we discuss below, it turns out that sensible results can be so obtained provided that one restricts to the regime of linear fluctuations about thermal equilibrium (which is all that is considered in [13,15, 16]), but this approach breaks down in general, as it does not respect the principles of hydrodynamic expansions.

Focussing first on the shortcomings of (2.15), we note that in the presence of a magnetic field there is a preferred rest frame, and in thermal equilibrium the fluid will be at rest in this frame. Since thermal states are not labeled by an arbitrary constant velocity $u^{\mu}$,

5 In this section we are only going to concern ourselves with the stress energy tensor since the conserved current is expressed in terms of the stress energy tensor by equation (2.2). 
there is no motivation for introducing a slowly varying velocity field as a hydrodynamical variable, which is the basis of (2.15). On the other hand, one can consider setting up an expansion in terms of the deviation of the fluid velocity from its equilibrium value, and this is effectively what is done in [13, 15, 16].

Even more problematic than the above is the fact that 2.15) implies incorrect relationships between transport coefficients due to an inappropriate imposition of Lorentz invariance where there is none. The presence of an external magnetic field explicitly breaks Lorentz invariance - there is now a preferred rest frame corresponding to the frame with pure B-field and no E-field. However, (2.15) fails to take this into account and is manifestly Lorentz covariant. Explicitly, the vector $v^{\mu}=\epsilon^{\mu \nu \rho} F_{\nu \rho}$ breaks the Lorentz symmetry, allowing us to separate out the $\mathrm{SO}(2)$ vector part $u^{i}$ of $u^{\mu}$ with a projection operator

$$
\tilde{\Delta}^{\mu \nu}=\eta^{\mu \nu}+\frac{v^{\mu} v^{\nu}}{|v|^{2}} .
$$

With this operator we can separate the transport coefficients according to their $\mathrm{SO}(2)$ representations. For example, if we look for the structure $u^{i} u^{j}$ in $T^{i j}$ and $u^{0} u^{0}$ in $T^{00}$, we see that both terms have coefficients fixed by the term $\epsilon u^{\mu} u^{\nu}$ in (2.15). However, if we use (2.17) to add additional terms to 2.15) of the form

$$
\tilde{\Delta}_{\alpha}^{\mu} \tilde{\Delta}_{\beta}^{\nu} u^{\alpha} u^{\beta}
$$

then the transport coefficient of $u^{i} u^{j}$ in $T^{i j}$ is now independent of the transport coefficient of $u^{0} u^{0}$.

Interestingly, the imposition of a spurious Lorentz invariance does not cause problems at the level of linearized fluctuations about thermal equilibrium. In the preferred frame, we write $u^{\mu}=v^{\mu}+\delta u^{\mu}$, with $\delta u^{0}=0$ and keep only terms linear in $\delta u^{i}$. To lowest order in this expansion $\Delta=\tilde{\Delta}$. Since the rest frame of the fluid very nearly corresponds with the preferred frame of the B-field, there is no difference between projecting linear fluctuations (or derivatives of fluctuations) to either the rest frame or the preferred frame since any difference will be second order. At any order in derivatives, $\mathrm{SO}(2)$ invariance lifts to lorentz invariance for linear fluctuations. This phenomena explains why other authors have not run into any trouble using (2.15): they were considering only linearized fluctuations. In order to study manifestly nonlinear properties though, we needed a new formalism. In particular, we avoid the problems described above by imposing only the actual $S O(2)$ symmetry, and using as hydrodynamical variables only those quantities that can be freely specified in equilibrium, namely the energy and charge density.

With the preceding comments in mind, it is now interesting to compare results obtained by these different approaches. To bridge the gap between the two formalisms, we define the fluid velocity in our formalism by equating the velocity with the Lorentz boost 
parameter needed to annihilate $T^{0 i}$. This definition is equivalent to matching (2.15) with (2.5) and reading off

$$
u^{i}=\frac{1}{\epsilon+P} T^{0 i}=\frac{2}{3 \epsilon}\left[\left(a_{1} \delta_{i j}+a_{2} \epsilon_{i j}\right) \partial_{j} \epsilon+\left(a_{3} \delta_{i j}+a_{4} \epsilon_{i j}\right) \partial_{j} \rho\right] .
$$

This expression is of course corrected at higher orders in the derivative expansion, but this version is sufficient for our current purposes. It is worth noting that this velocity is manifestly small in the hydrodynamic expansion regardless of the amplitude of any fluctuations as it is proportional to the derivatives of our charge and energy densities.

With our definition of the velocity in hand, we can now use (2.15) to find a suitable definition for the shear viscosity. Since (2.15) is valid for linear fluctuations, we can use (2.19) as a dictionary to relate the shear viscosity $\eta$ to our linear transport coefficients. If we plug (2.19) into (2.15) and keep only terms linear in our fluctuations then we have

$$
T^{(2) i j}=\frac{4 \eta}{3}\left[\left(a_{1} \delta_{i k}+a_{2} \epsilon_{i k}\right) \partial_{i} \partial_{k} \epsilon+\left(a_{3} \delta_{i k}+a_{4} \epsilon_{i k}\right) \partial_{i} \partial_{k} \rho\right]^{s t} .
$$

Note that a first order contribution in a hydrodynamic expansion with respect to the velocities is equivalent here to a second order contribution with respect to the charge and energy densities since the velocity itself is now being considered first order in the hydrodynamic expansion. As noted above, second order linear contributions to the stress tensor from derivatives of the charge density were not included in (2.15), so the second half of (2.20) is not predictive of any relationship with our current results. This means that we should only interpret the shear viscosity as a factor in the diffusion of energy and not charge. Since the only terms contributing to the second order linear energy transport coefficients from the standard formalism are those in (2.20), we can read off relationships between our transport coefficients $b_{1}^{\epsilon}, b_{2}^{\epsilon}$ and the shear viscosity $\eta$. Comparison with (2.8) yields

$$
b_{1}^{\epsilon}=\frac{4 \eta a_{1}}{3}, \quad b_{2}^{\epsilon}=\frac{4 \eta a_{2}}{3} .
$$

If we skip ahead and insert the values for these transport coefficients that we obtain from AdS/CFT in section 6, we see that both of these equations imply that

$$
\eta=\frac{a^{2}}{4}
$$

where $a$ is related to the entropy density $s$ according to $s=\pi a^{2}$. Putting these facts together yields the universal ratio

$$
\frac{\eta}{s}=\frac{1}{4 \pi} .
$$

This result is a slight extension of [16] which demonstrated this result for uncharged fluids in the presence of a magnetic field. It should be noted however that while our result takes 
into account additional degrees of freedom in the form of a variable charge density, we have not used any nonlinear effects to demonstrate this relationship. As noted above, the shear viscosity is a purely linear property of our system and has no dependence on nonlinear properties.

\section{Gravity side}

We now turn to the gravitational description of fluid dynamics. In this section we write down the dyonic black brane solution and its thermodynamic properties, and give the general prescription for computing the boundary stress tensor and current.

\subsection{Action and dyonic black brane solution}

The bulk Maxwell-Einstein action with a negative cosmological constant, which can be thought of as a consistent truncation of the theory resulting from an $S^{7}$ compactification of M-theory, is

$$
S=\frac{2}{\kappa_{4}^{2}} \int d^{4} x \sqrt{-g}\left[\frac{1}{4} R-\frac{1}{4} F_{M N} F^{M N}-\frac{3}{2 L^{2}}\right] .
$$

We henceforth choose units with $L=1$. The equations of motion are

$$
\begin{aligned}
W_{M N} & \equiv R_{M N}+3 g_{M N}-2 F_{M P} F_{N}^{P}+\frac{1}{2} g_{M N} F_{P Q} F^{P Q}=0 \\
Y^{N} & \equiv \nabla_{M} F^{M N}=0
\end{aligned}
$$

The dyonic black brane solution in Eddington-Finkelstein coordinates is

$$
\begin{aligned}
d s^{2} & =2 d v d r-U(r) d v^{2}+r^{2} d x^{i} d x^{i} \\
F & =\frac{\rho}{r^{2}} d r \wedge d v+B d x^{1} \wedge d x^{2}
\end{aligned}
$$

where $\mathrm{U}(\mathrm{r})$ is given by

$$
U(r)=r^{2}+\frac{\rho^{2}+B^{2}}{r^{2}}-\frac{2 \epsilon}{r} .
$$

The horizon is located at the largest real root of the equation $U(r)=0$. Calling this $r=a$, $a$ is thus given by the largest real root of

$$
a^{2}+\frac{\rho^{2}+B^{2}}{a^{2}}-\frac{2 \epsilon}{a}=0
$$

The energy and charge densities (defined below) are given by $\epsilon$ and $\rho$.

The Hawking temperature is

$$
T=\frac{3 a}{4 \pi}-\frac{B^{2}+\rho^{2}}{4 \pi a^{3}} .
$$


$\epsilon$ and $\rho$ are restricted to values such that $T \geq 0$. The chemical potential can be read off from the asymptotic value of $A_{v}$ in a gauge such that $A_{v}$ vanishes at the horizon. This gives $\mu=\frac{\rho}{a}$.

Some further conventions: Latin indices $M, N, \ldots$ run over all four spacetime coordinates, while Greek indices $\mu, \nu, \ldots$ run over the three coordinates $\left(v, x^{1}, x^{2}\right)$. Since $v$ plays the role of time on the boundary, we will sometimes use $v=x^{0}$. The boundary theory will always see a Minkowski metric, $\tilde{\gamma}_{\mu \nu} d x^{\mu} d x^{\nu}=-\left(d x^{0}\right)^{2}+d x^{i} d x^{i}$. Indices on the boundary stress tensor and currents are raised and lowered with this metric.

\subsection{Stress tensor and current}

The action (3.1) should be supplemented with the boundary terms

$$
S_{b n d y}=-\frac{1}{\kappa_{4}^{2}} \int_{\partial M} d^{3} x \sqrt{-\gamma} \theta-\frac{2}{\kappa_{4}^{2}} \int_{\partial M} d^{3} x \sqrt{-\gamma} .
$$

Here $\gamma$ is the boundary metric and $\theta=\gamma^{\mu \nu} \theta_{\mu \nu}$, where $\theta_{\mu \nu}=-\frac{1}{2}\left(\nabla_{\mu} n_{\nu}+\nabla_{\nu} n_{\mu}\right)$ is the extrinsic curvature of the boundary, defined in terms of the outward pointing unit normal vector $n$.

The conformal boundary metric is defined as $\tilde{\gamma}_{\mu \nu}=\lim _{r \rightarrow \infty} \frac{1}{r^{2}} \gamma_{\mu \nu}$. Also, the boundary gauge field is defined as $\lim _{r \rightarrow \infty} A_{\mu}$, in a gauge where $n^{M} A_{M}=0$. The boundary stress tensor and current are then defined as

$$
\delta S=\frac{1}{\kappa_{4}^{2}} \int_{\partial M} \sqrt{-\tilde{\gamma}}\left(2 J^{\mu} \delta A_{\mu}+T^{\mu \nu} \delta \tilde{\gamma}_{\mu \nu}\right)
$$

Explicitly [17,

$$
\begin{aligned}
J^{\mu} & =r^{2} F^{\mu r} \\
T^{\mu \nu} & =\frac{r^{5}}{2}\left[\theta^{\mu \nu}-\theta \gamma^{\mu \nu}-2 \gamma^{\mu \nu}\right] .
\end{aligned}
$$

Implicit in (3.9) is the large $r$ limit, as well as a projection of $T^{\mu \nu}$ parallel to the boundary (since the orthogonal component does not appear in (3.8).)

Electromagnetic gauge invariance implies current conservation,

$$
\nabla_{\mu} J^{\mu}=0
$$

Invariance under diffeomorphisms generated by vector fields tangent to the boundary yields the (non) conservation equation

$$
\nabla_{\nu} T^{\mu \nu}=F^{\mu \nu} J_{\nu}
$$


Tracelessness of the stress tensor follows from invariance under diffeomorphisms shifting the radial location of the boundary

$$
\tilde{\gamma}_{\mu \nu} T^{\mu \nu}=0
$$

In particular, the latter invariance follows from the absence of logarithmic divergences in the bulk action, the presence of which would necessitate adding a non-diff invariant counterterm [18].

Applied to the solution (3.3) we find

$$
\begin{aligned}
T^{\mu \nu} & =\operatorname{diag}\left(\epsilon, \frac{1}{2} \epsilon, \frac{1}{2} \epsilon\right) \\
J^{\mu} & =(\rho, 0,0) .
\end{aligned}
$$

\section{Gravitational derivative expansion}

The dyonic black brane solution above corresponds to a fluid in thermal equilibrium. To find a solution corresponding to local thermal equilibrium, we follow the logic in [1] and construct a solution which looks locally like the dyonic black brane. We take the expression in (3.3) and allow $\epsilon$ and $\rho$ to be functions of the boundary coordinates $x^{\mu}$. While this expression is no longer a solution of the Maxwell-Einstein equations, it is approximately a solution in the limit that derivatives of $\epsilon$ and $\rho$ are small. By adding small corrections order by order in a derivative expansion we construct a perturbative solution which locally approximates the dyonic black hole.

To implement the derivative expansion, we formally regard $\epsilon$ and $\rho$ as being functions of $\varepsilon x^{\mu}$, where $\varepsilon$ is a formal expansion parameter that is eventually set equal to 1 . The metric and gauge fields are expanded as

$$
\begin{aligned}
& g=g^{(0)}(\epsilon, \rho)+\varepsilon g^{(1)}(\epsilon, \rho)+\varepsilon^{2} g^{(2)}(\epsilon, \rho)+\mathcal{O}\left(\varepsilon^{3}\right) \\
& A=A^{(0)}(\epsilon, \rho)+\varepsilon A^{(1)}(\epsilon, \rho)+\varepsilon^{2} A^{(2)}(\epsilon, \rho)+\mathcal{O}\left(\varepsilon^{3}\right),
\end{aligned}
$$

where $g^{(0)}$ and $A^{(0)}$ represent the lowest order solution given in (3.3). The energy and charge densities are themselves given by an expansion,

$$
\epsilon=\epsilon^{(0)}\left(\varepsilon x^{\mu}\right)+\varepsilon \epsilon^{(1)}\left(\varepsilon x^{\mu}\right)+\cdots, \quad \rho=\rho^{(0)}\left(\varepsilon x^{\mu}\right)+\varepsilon \rho^{(1)}\left(\varepsilon x^{\mu}\right)+\cdots
$$

As explained in [1], the equations of motion can be solved "tubewise" by working near a given $x^{\mu}$ location, say $x^{\mu}=0$. It is then convenient to set $\epsilon^{(n>0)}(0)=\rho^{(n>0)}(0)=0$. In the following, the order of a quantity is defined with respect to its associated power of $\varepsilon$. Note that this is slightly different than the labelling used in section 2 where we just counted the number of derivatives acting on $\epsilon$ and $\rho$. 
For the metric and gauge fields we make the gauge choice

$$
A_{r}=0, \quad g_{r r}=0, \quad g^{(0)^{\mu \nu}} g_{\mu \nu}^{(n>0)}=0 .
$$

The zeroth order solution preserves $S O(2)$ rotational symmetry, and this can be used to classify the corrections to the metric and gauge fields. The gauge field corrections are written

$$
A^{(n)}=A_{v}^{(n)} d v+A_{i}^{(n)} d x^{i},
$$

with $A_{v}^{(n)}$ an $S O(2)$ scalar and $A_{i}^{(n)}$ an $S O(2)$ vector. For the metric we write

$$
\left(d s^{2}\right)^{(n)}=\frac{k^{(n)}}{r^{2}} d v^{2}-2 h^{(n)} d v d r+r^{2} h^{(n)} d x^{i} d x^{i}+2 j_{i}^{(n)} d v d x^{i}+r^{2} \sigma_{i j}^{(n)} d x^{i} d x^{j} .
$$

In this expansion, $k^{(n)}$ and $h^{(n)}$ are $S O(2)$ scalars; $j_{i}^{(n)}$ is an $S O(2)$ vector; and $\sigma_{i j}^{(n)}$ is an $S O(2)$ symmetric traceless tensor.

We impose the following large $r$ boundary conditions on the $n>0$ components:

$$
\begin{aligned}
& A_{v}^{(n)} \sim \frac{1}{r^{2}}, \quad A_{i}^{(n)} \sim \frac{1}{r} \\
& k^{(n)} \sim r^{0} \quad h^{(n)} \sim \frac{1}{r^{4}}, \quad j_{i}^{(n)} \sim \frac{1}{r}, \quad \sigma_{i j}^{(n)} \sim \frac{1}{r^{3}} .
\end{aligned}
$$

These conditions follow from a combination of the asymptotic AdS boundary conditions along with the freedom to redefine coordinates as well as the zeroth order solution, as in [3].

In addition to these large $r$ boundary conditions, we must also demand that our solution be smooth across the horizon at $r=a$ in order to uniquely determine a solution. As shown in [19], this condition is equivalent to demanding the presence of purely ingoing modes at the future horizon.

Although the Maxwell-Einstein equations of motion are invariant under electricmagnetic duality, the boundary conditions (4.6) are not. In particular, our boundary conditions fix a constant magnetic field and vanishing electric field on the boundary. However, electric-magnetic duality will turn on a nonzero electric field in general. See [20] for further discussion of the breaking of duality by AdS/CFT boundary conditions.

We then find the current and stress tensor (a large $r$ limit is implicit):

$$
\begin{aligned}
J^{v} & =\rho \\
J^{i} & =-r^{2} \sum_{n} \partial_{r} A_{i}^{(n)} \\
T^{v v} & =\epsilon \\
T^{v i} & =-\frac{3}{4} r \sum_{n} j_{i}^{(n)} \\
T^{i j} & =\frac{1}{2} \epsilon+\frac{3}{4} r^{3} \sum_{n} \sigma_{i j}^{(n)}
\end{aligned}
$$




\section{Structure of perturbation theory}

The $n$th order metric coefficients are determined by the components of the Einstein equations $W_{M N}^{(n)}=0$ with $M, N \neq v$. These equations can be organized as

$$
\begin{aligned}
W_{r r}^{(n)} & =-\frac{1}{r^{4}} \partial_{r}\left(r^{4} \partial_{r} h^{(n)}\right)-S_{(h)}^{(n)}=0 \\
r^{2}\left(U W_{r r}\right)^{(n)}-W_{i i}^{(n)} & =\partial_{r}\left(-\frac{2}{r} k^{(n)}\right)+\partial_{r}\left(\partial_{r}\left(r^{2} U^{(0)}\right) h^{(n)}\right)-\frac{8}{r^{2}} B^{2} h^{(n)}+4 \rho^{(0)} \partial_{r} A_{v}^{(n)}-S_{(k)}^{(n)}=0 \\
W_{r i}^{(n)} & =\frac{1}{2} r \partial_{r}\left(\frac{1}{r^{2}} \partial_{r}\left(r j_{i}^{(n)}\right)\right)+\frac{2}{r^{2}}\left[\rho^{(0)} \delta_{i j}-B \epsilon_{i j}\right] \partial_{r} A_{j}^{(n)}-S_{i}^{(n)}=0 \\
W_{i j}^{(n)}-\frac{1}{2} \delta_{i j} W_{k k}^{(n)} & =\partial_{r}\left(-\frac{1}{2} r^{2} U^{(0)} \partial_{r} \sigma_{i j}^{(n)}\right)-S_{i j}^{(n)}=0
\end{aligned}
$$

The source terms appearing above are constructed from the solution at order $n-1$, and so are assumed to be known.

Similarly, two components of the Maxwell equations yield

$$
\begin{aligned}
Y^{(n) v} & =\frac{1}{r^{2}} \partial_{r}\left(-r^{2} \partial_{r} A_{v}^{(n)}-2 \rho^{(0)} h^{(n)}\right)-V^{(n)}=0 \\
Y^{(n) i} & =\frac{1}{r^{2}} \partial_{r}\left(U^{(0)} \partial_{r} A_{i}^{(n)}+\frac{1}{r^{2}}\left[\rho^{(0)} \delta_{i j}+B \epsilon_{i j}\right] j_{j}^{(n)}\right)-V_{i}^{(n)}=0
\end{aligned}
$$

These equations, together with the boundary conditions (4.6), are sufficient information to solve for all the metric and gauge field functions in terms of $\epsilon$ and $\rho$. The remaining Einstein-Maxwell equations then becomes conditions on $\epsilon$ and $\rho$; in particular, they are identified with equations of fluid dynamics (1.1) expanded to a given order in $\varepsilon$.

\section{Solving the equations}

\subsection{Zeroth order solution}

At zeroth order we use the solution (3.3) but with $\epsilon=\epsilon^{(0)}$ and $\rho=\rho^{(0)}$. By construction, this is a solution to the Einstein-Maxwell equations. The current and stress tensor are

$$
\begin{aligned}
J^{\mu} & =(\rho, 0,0) \\
T^{\mu \nu} & =\operatorname{diag}\left(\epsilon, \frac{1}{2} \epsilon, \frac{1}{2} \epsilon\right) .
\end{aligned}
$$

\subsection{First order solution}

At first order we write

$$
\begin{aligned}
& \epsilon\left(x^{\mu}\right)=\epsilon^{(0)}+\varepsilon x^{\mu} \partial_{\mu} \epsilon^{(0)}(0) \\
& \rho\left(x^{\mu}\right)=\rho^{(0)}+\varepsilon x^{\mu} \partial_{\mu} \rho^{(0)}(0) .
\end{aligned}
$$


The first order sources are built out of $\partial_{\mu} \epsilon^{(0)}$ and $\partial_{\mu} \rho^{(0)}$, and read

$$
\begin{aligned}
S_{(h)}^{(1)} & =S_{(k)}^{(1)}=S_{i}^{(1)}=S_{i j}^{(1)}=V^{(1)}=0, \\
V_{i}^{(1)} & =\frac{\partial_{i} \rho^{(0)}}{r^{4}}
\end{aligned}
$$

All of the functions in (5.1)-(5.2) appearing without sources can be set to zero, either due to the boundary conditions or by absorbing into the lowest order solution. What remains is to find $\partial_{r} A_{i}^{(1)}$ and $j_{i}^{(1)}$.

Integrating $Y^{(1) i}=0$ gives

$$
U^{(0)} \partial_{r} A_{i}^{(1)}+\frac{1}{r^{2}}\left[\rho^{(0)} \delta_{i j}+B \epsilon_{i j}\right] j_{j}^{(1)}+\frac{\partial_{i} \rho^{(0)}}{r}=-c_{i}^{(1)}
$$

where $c_{i}$ is $r$-independent. Solving for $\partial_{r} A_{i}^{(1)}$ and plugging into $W_{r i}^{(1)}=0$ gives

$$
U^{(0)} \partial_{r}^{2} j_{i}^{(1)}-U^{\prime \prime(0)} j_{i}^{(1)}=\frac{4}{r^{2}}\left(\rho^{(0)} \delta_{i j}-B \epsilon_{i j}\right)\left(c_{j}^{(1)}+\frac{1}{r} \partial_{j} \rho^{(0)}\right)
$$

where' denotes the radial derivative and we used the following identity for $U$ :

$$
U^{\prime \prime}=\frac{2 U}{r^{2}}+\frac{4}{r^{2}}\left(\rho^{2}+B^{2}\right)
$$

The general solution of $(6.5)$ is

$$
j_{i}^{(1)}(r)=-U^{(0)}(r)\left(\rho^{(0)} \delta_{i j}-B \epsilon_{i j}\right) \int_{\alpha_{j}^{(1)}}^{r} d r^{\prime} \frac{\beta_{j}^{(1)}+\frac{4}{r^{\prime}} c_{j}^{(1)}+\frac{2}{r^{\prime 2}} \partial_{j} \rho^{(0)}}{U^{(0)}\left(r^{\prime}\right)^{2}},
$$

with integration constants $\alpha_{j}^{(1)}$ and $\beta_{j}^{(1)}$. The integral is elementary but lengthy — see the appendix. The large $r$ boundary condition fixes $\alpha_{j}^{(1)}=\infty \cdot \beta_{j}^{(1)}$ is fixed by demanding regularity at the horizon. In particular, for generic $\beta_{j}^{(1)}$ we find that $j_{i}^{(1)}$ has a logarithmic term, $j_{i}^{(1)} \sim(r-a) \ln (r-a)$, which yields a divergent derivative. We can cancel this term by choosing

$$
\beta_{i}^{(1)}=-\frac{4}{a^{(0)}}\left(1+\frac{U^{\prime(0)}\left(a^{(0)}\right)}{a^{(0)} U^{\prime \prime(0)}\left(a^{(0)}\right)}\right) c_{i}^{(1)}-\frac{2}{a^{(0)^{2}}}\left(1+\frac{2 U^{\prime(0)}\left(a^{(0)}\right)}{a^{(0)} U^{\prime \prime(0)}\left(a^{(0)}\right)}\right) \partial_{i} \rho^{(0)} .
$$

With this choice of integration constants we find that $j_{i}^{(1)}$ has the large $r$ behavior

$$
j_{i}^{(1)}(r)=\frac{\left(\rho^{(0)} \delta_{i j}-B \epsilon_{i j}\right) \beta_{j}^{(1)}}{3 r}+\mathcal{O}\left(\frac{1}{r^{2}}\right)
$$


$\partial_{r} A_{i}^{(1)}$ can now be found from (6.4).

Up to first order the current and stress tensor can now be computed as

$$
\begin{aligned}
& J^{i}=c_{i}^{(1)} \\
& T^{v i}=-\frac{1}{4}\left(\rho^{(0)} \delta_{i j}-B \epsilon_{i j}\right) \beta_{j}^{(1)} \\
& {\left[T^{i j}\right]^{s t}=0 .}
\end{aligned}
$$

The remaining Maxwell-Einstein equations are $W_{v M}^{(1)}=0$ and $Y^{(1) r}=0$. With the above expressions for $j_{i}^{(1)}$ and $\partial_{r} A_{i}^{(1)}$ we can verify that these equations are equivalent to the order $\varepsilon$ part of the equations

$$
\partial_{\mu} J^{\mu}=0, \quad \partial_{\nu} T^{\mu \nu}=F^{\mu \nu} J_{\nu} .
$$

Note that for the powers of $\varepsilon$ to match up, the stress tensor and current appearing with a derivative are given by their lowest order expressions (6.1), while the undifferentiated current is given by (6.10). In particular, these equations imply

$$
\begin{gathered}
\partial_{v} \rho^{(0)}=\partial_{v} \epsilon^{(0)}=0 \\
c_{i}^{(1)}=-\frac{1}{2 B} \epsilon_{i j} \partial_{j} \epsilon^{(0)} .
\end{gathered}
$$

We can now read off the first order transport coefficients by writing

$$
T^{v i}=\left(a_{1} \delta_{i j}+a_{2} \epsilon_{i j}\right) \partial_{j} \epsilon+\left(a_{3} \delta_{i j}+a_{4} \epsilon_{i j}\right) \partial_{j} \rho,
$$

as in (2.5). Using the explicit form of $U$ as well as the relation between $\epsilon$ and $a$, we find

$$
\begin{aligned}
& a_{1}=-\frac{3}{4}\left(\frac{\epsilon}{\rho^{2}+B^{2}}\right) \\
& a_{2}=-\frac{3}{4}\left(\frac{\epsilon}{\rho^{2}+B^{2}}\right) \frac{\rho}{B} \\
& a_{3}=\frac{1}{2}\left(\frac{\rho}{\rho^{2}+B^{2}}\right)\left(\frac{3 a \epsilon-\rho^{2}-B^{2}}{a^{2}}\right) \\
& a_{4}=-\frac{1}{2}\left(\frac{B}{\rho^{2}+B^{2}}\right)\left(\frac{3 a \epsilon-\rho^{2}-B^{2}}{a^{2}}\right) .
\end{aligned}
$$

\subsection{Second order solution}

$\epsilon$ and $\rho$ are now given by expanding out (4.2) to order $\varepsilon^{2}$,

$$
\begin{aligned}
& \epsilon\left(x^{\mu}\right)=\epsilon^{(0)}(0)+\varepsilon x^{\mu} \partial_{\mu} \epsilon^{(0)}(0)+\frac{1}{2} \varepsilon^{2} x^{\mu} x^{\nu} \partial_{\mu} \partial_{\nu} \epsilon^{(0)}(0)+\varepsilon^{2} x^{\mu} \partial_{\mu} \epsilon^{(1)}(0) \\
& \rho\left(x^{\mu}\right)=\rho^{(0)}(0)+\varepsilon x^{\mu} \partial_{\mu} \rho^{(0)}(0)+\frac{1}{2} \varepsilon^{2} x^{\mu} x^{\nu} \partial_{\mu} \partial_{\nu} \rho^{(0)}(0)+\varepsilon^{2} x^{\mu} \partial_{\mu} \rho^{(1)}(0) .
\end{aligned}
$$


The second order sources work out to be

$$
\begin{aligned}
S_{(h)}^{(2)}= & \frac{2}{r^{2}}\left(\partial_{r} A_{i}^{(1)}\right)^{2} \\
S_{(k)}^{(2)}= & 2 U^{(0)}\left(\partial_{r} A_{i}^{(1)}\right)^{2}+\frac{4}{r^{2}} \rho^{(0)} \partial_{r} A_{i}^{(1)} j_{i}^{(1)}-\frac{4}{r^{2}} B \epsilon_{i j} \partial_{i} A_{j}^{(1)} \\
& +\frac{2}{r} \partial_{i} j_{i}^{(1)}+\partial_{r} \partial_{i} j_{i}^{(1)}-\frac{2}{r} j_{i}^{(1)} \partial_{r} j_{i}^{(1)}-\frac{1}{2}\left(\partial_{r} j_{i}^{(1)}\right)^{2} \\
S_{i}^{(2)}= & 0 \\
S_{i j}^{(2)}= & -\partial_{r}\left[\partial_{i} j_{j}^{(1)}\right]^{s t}-\frac{2}{r}\left[j_{i}^{(1)} \partial_{r} j_{j}^{(1)}\right]^{s t}+\frac{2}{r^{2}}\left[j_{i}^{(1)} j_{j}^{(1)}\right]^{s t}+\frac{1}{2}\left[\partial_{r} j_{i}^{(1)} \partial_{r} j_{j}^{(1)}\right]^{s t} \\
+ & 2 U^{(0)}\left[\partial_{r} A_{i}^{(1)} \partial_{r} A_{j}^{(1)}\right]^{s t}+\frac{4}{r}\left[\partial_{i} \rho^{(0)} \partial_{r} A_{j}^{(1)}\right]^{s t}+\frac{4}{r^{2}} B\left[\partial_{r} A_{i}^{(1)} \epsilon_{j k} j_{k}^{(1)}\right]^{s t} \\
V^{(2)}= & \frac{1}{r^{2}} \partial_{r} \partial_{i} A_{i}^{(1)}-\frac{1}{r^{2}} \partial_{r}\left(j_{i}^{(1)} \partial_{r} A_{i}^{(1)}\right) \\
V_{i}^{(2)}= & 0 .
\end{aligned}
$$

We can solve the equations separately in the scalar, vector, and symmetric traceless tensor sectors.

\section{$\underline{\text { scalar sector : }}$}

We determine $h^{(2)}$ from the equation $W_{r r}^{(2)}=0$, which takes the form

$$
\partial_{r}\left(r^{4} \partial_{r} h_{2}\right)=-2 r^{2}\left(\partial_{r} A_{i}^{(1)}\right)^{2} .
$$

The source on the right hand side is determined from (6.4), (6.7), and (6.12). Of main relevance is the fact that $\partial_{r} A_{i}^{(1)}$ is a smooth function, including at the horizon, and has asymptotic behavior

$$
\partial_{r} A_{i}^{(1)}=-\frac{c_{i}^{(1)}}{r^{2}}+\mathcal{O}\left(\frac{1}{r^{3}}\right) .
$$

We can therefore proceed by straightforward integration:

$$
h^{(2)}(r)=-2 \int_{\infty}^{r} \frac{d r^{\prime}}{r^{\prime 4}} \int_{\infty}^{r^{\prime}} d r^{\prime \prime}\left(r^{\prime \prime} \partial_{r} A_{i}^{(1)}\left(r^{\prime \prime}\right)\right)^{2} .
$$

Next, $\partial_{r} A_{v}^{(2)}$ is found from integrating $Y^{(2) v}=0$. Writing this equation as $\frac{1}{r^{2}} \partial_{r}\left(-r^{2} \partial_{r} A_{v}^{(2)}\right)+X_{1}=0$, the solution with the required boundary conditions is

$$
\partial_{r} A_{v}^{(2)}(r)=\frac{1}{r^{2}} \int_{\infty}^{r} d r^{\prime} r^{\prime 2} X_{1}\left(r^{\prime}\right)
$$

Finally, $k^{(2)}$ is found by integrating $r^{2}\left(U W_{r r}\right)^{(2)}-W_{i i}^{(2)}=0$. Writing this equation in the form $\partial_{r}\left(-\frac{2}{r} k^{(2)}\right)+X_{2}=0$ we can straightforwardly integrate to get

$$
k^{(2)}(r)=\frac{1}{2} r \int_{\infty}^{r} d r^{\prime} X_{2}\left(r^{\prime}\right)
$$


This completes the solution in the scalar sector. The explicit integrals appearing above are complicated and not especially illuminating. Since these functions do not show up in the stress tensor and current it is not necessary to compute them explicitly in order to find the second order transport coefficients.

\section{vector sector :}

The vector sector is simple since the corresponding source terms in (6.16) are zero. The solution is obtained as in the first order computation, with the only difference that we do not have the analog of the source terms proportional to $\partial_{i} \rho^{(0)}$. We thus have

$$
j_{i}^{(2)}(r)=-U^{(0)}(r)\left(\rho^{(0)} \delta_{i j}-B \epsilon_{i j}\right) \int_{\infty}^{r} d r^{\prime} \frac{\beta_{j}^{(2)}+\frac{4}{r^{\prime}} c_{j}^{(2)}}{U^{(0)}\left(r^{\prime}\right)^{2}},
$$

with

$$
\beta_{i}^{(2)}=-\frac{4}{a^{(0)}}\left(1+\frac{U^{\prime(0)}\left(a^{(0)}\right)}{a^{(0)} U^{\prime \prime(0)}\left(a^{(0)}\right)}\right) c_{i}^{(2)} .
$$

$\partial_{r} A_{i}^{(2)}$ is determined by the analog of (6.4),

$$
U^{(0)} \partial_{r} A_{i}^{(2)}+\frac{1}{r^{2}}\left[\rho^{(0)} \delta_{i j}+B \epsilon_{i j}\right] j_{j}^{(2)}=-c_{i}^{(2)} .
$$

$\beta_{i}^{(2)}$ and $c_{i}^{(2)}$ now contribute to the stress tensor and current precisely as in (6.10). Also, the stress tensor conservation equation now fixes

$$
c_{i}^{(2)}=-\frac{1}{2 B} \epsilon_{i j} \partial_{j} \epsilon^{(1)} .
$$

just as in (6.12). Therefore, the vector components of the stress tensor and current at this order take the same form as at first order,

$$
\begin{aligned}
& J^{i}=-\frac{1}{2 B} \epsilon_{i j} \partial_{j} \epsilon \\
& T^{v i}=\left(a_{1} \delta_{i j}+a_{2} \epsilon_{i j}\right) \partial_{j} \epsilon+\left(a_{3} \delta_{i j}+a_{4} \epsilon_{i j}\right) \partial_{j} \rho .
\end{aligned}
$$

with the same coefficients (6.14). In writing this expression for $T^{v i}$ we have used $\rho^{(1)}\left(x^{\mu}\right)=$ 0 . This is consistent, since the equation $\partial_{\mu} J^{\mu}=0$ at order $\varepsilon^{2}$ is simply $\partial_{v} \rho^{(1)}=0$, as follows from the fact that the spatial current is divergenceless at this order.

This completes the solution in the vector sector.

\section{$\underline{\text { tensor sector: }}$}

The tensor components are determined by solving $W_{i j}^{(2)}-\frac{1}{2} \delta_{i j} W_{k k}^{(2)}=0$. Integrating, we obtain

$$
\sigma_{i j}^{(2)}(r)=-2 \int_{\infty}^{r} \frac{d r^{\prime}}{r^{\prime 2} U^{(0)}\left(r^{\prime}\right)} \int_{a^{(0)}}^{r^{\prime}} d r^{\prime \prime} S_{i j}^{(2)}\left(r^{\prime \prime}\right) .
$$


The lower integration limit on $r^{\prime \prime}$ is chosen to avoid a singularity at the horizon, while the integration limit for $r^{\prime}$ is chosen to impose the correct asymptotic behavior.

Of primary interest is the contribution to $T^{i j}$ given in (4.7). This gives

$$
\left[T^{i j}\right]^{s t}=\frac{1}{2} \int_{a^{(0)}}^{\infty} d r S_{i j}^{(2)}(r)
$$

From this we can read off transport coefficients $b_{1,2,3,4}$ appearing in (2.8).

The simplest contributions are the second derivative terms, $b_{1}^{a}\left[\partial_{i} \partial_{j} q_{a}\right]$ and $\left.b_{2}^{a}\left[\epsilon_{i k} \partial_{k} \partial_{j} q_{a}\right]\right]^{s t}$, which only get a contribution from the source term $S_{i j}^{(2)}=-\partial_{r}\left[\partial_{i} j_{j}^{(1)}\right]^{s t}$. We find

$$
\begin{aligned}
& b_{1}^{\epsilon}=\frac{1}{a^{(0)^{2}} U^{\prime \prime(0)}\left(a^{(0)}\right)}=\frac{1}{4} \frac{a^{2}}{\left(\rho^{2}+B^{2}\right)} \\
& b_{1}^{\rho}=-\frac{2 \rho^{(0)}}{a^{(0)^{3}} U^{\prime \prime(0)}\left(a^{(0)}\right)}=-\frac{1}{2} \frac{a \rho}{\left(\rho^{2}+B^{2}\right)} \\
& b_{2}^{\epsilon}=\frac{\rho^{(0)}}{a^{(0)^{2}} U^{\prime \prime(0)}\left(a^{(0)}\right) B}=\frac{1}{4} \frac{a^{2}}{\left(\rho^{2}+B^{2}\right)} \frac{\rho}{B} \\
& b_{2}^{\rho}=\frac{2 B}{a^{(0)^{3}} U^{\prime \prime(0)}\left(a^{(0)}\right)}=\frac{1}{2} \frac{a B}{\left(\rho^{2}+B^{2}\right)} \text {. }
\end{aligned}
$$

The remaining nonlinear transport coefficients are much more tedious to obtain. There are two contributions to the stress tensor: $\left[T^{i j}\right]^{s t}=\left[T_{1}^{i j}\right]^{s t}+\left[T_{2}^{i j}\right]^{s t}$.

The contribution from the source term $S_{i j}^{(2)}=-\partial_{r}\left[\partial_{i} j_{j}^{(1)}\right]^{s t}$ is easy to obtain, and yields the stress tensor

$$
\begin{aligned}
{\left[T_{1}^{i j}\right]^{s t} } & =\frac{1}{4} \frac{\partial}{\partial \epsilon}\left(\frac{a(\epsilon, \rho)^{2}}{\rho^{2}+B^{2}}\right)\left[\partial_{i} \epsilon \partial_{j} \epsilon\right]^{s t}-\frac{1}{2} \frac{\partial}{\partial \rho}\left(\frac{a(\epsilon, \rho) \rho}{\rho^{2}+B^{2}}\right)\left[\partial_{i} \rho \partial_{j} \rho\right]^{s t} \\
& +\left[\frac{1}{4} \frac{\partial}{\partial \rho}\left(\frac{a(\epsilon, \rho)^{2}}{\rho^{2}+B^{2}}\right)-\frac{1}{2} \frac{\partial}{\partial \epsilon}\left(\frac{a(\epsilon, \rho) \rho}{\rho^{2}+B^{2}}\right)\right]\left[\partial_{i} \epsilon \partial_{j} \rho\right]^{s t} \\
& +\frac{1}{4 B} \frac{\partial}{\partial \epsilon}\left(\frac{a(\epsilon, \rho)^{2} \rho}{\rho^{2}+B^{2}}\right)\left[\epsilon_{i k} \partial_{k} \epsilon \partial_{j} \epsilon\right]^{s t}+\frac{B}{2} \frac{\partial}{\partial \rho}\left(\frac{a(\epsilon, \rho)}{\rho^{2}+B^{2}}\right)\left[\epsilon_{i k} \partial_{k} \rho \partial_{j} \rho\right]^{s t} \\
& +\left[\frac{1}{4 B} \frac{\partial}{\partial \rho}\left(\frac{a(\epsilon, \rho)^{2} \rho}{\rho^{2}+B^{2}}\right)+\frac{B}{2} \frac{\partial}{\partial \epsilon}\left(\frac{a(\epsilon, \rho)}{\rho^{2}+B^{2}}\right)\right]\left[\epsilon_{i k} \partial_{k} \epsilon \partial_{j} \rho\right]^{s t} .
\end{aligned}
$$

Here $a(\epsilon, \rho)$ is obtained by solving (3.5).

The rest of the contribution $T_{2}^{i j}$ take the form of integrals, which we will not evaluate explicitly. The sources are built up out of $j_{i}^{(1)}$ and $\partial_{r} A_{i}^{(1)}$. These were found in the first order analysis, and we write them in the form

$$
\begin{aligned}
j_{i}^{(1)} & =\left(\rho \delta_{i j}-B \epsilon_{i j}\right)\left(j_{\epsilon}(r) \epsilon_{j k} \partial_{k} \epsilon+j_{\rho}(r) \partial_{j} \rho\right) \\
\partial_{r} A_{i}^{(1)} & =A_{\epsilon}(r) \epsilon_{i j} \partial_{j} \epsilon+A_{\rho}(r) \partial_{i} \rho
\end{aligned}
$$


with

$$
\begin{aligned}
& j_{\epsilon}(r)=\frac{2}{B a} U(r) \int_{\infty}^{r} d r^{\prime}\left(\frac{1+\frac{U^{\prime}(a)}{a U^{\prime \prime}(a)}-\frac{a}{r^{\prime}}}{U\left(r^{\prime}\right)^{2}}\right) \\
& j_{\rho}(r)=\frac{2}{a^{2}} U(r) \int_{\infty}^{r} d r^{\prime}\left(\frac{1+\frac{2 U^{\prime}(a)}{a U^{\prime \prime}(a)}-\frac{a^{2}}{r^{\prime 2}}}{U\left(r^{\prime}\right)^{2}}\right) \\
& A_{\epsilon}(r)=-\frac{1}{2 B U(r)}-\frac{1}{4} \frac{a^{4} U^{\prime \prime}(a)}{r^{2} U(r)} j_{\epsilon}(r) \\
& A_{\rho}(r)=-\frac{1}{r U(r)}-\frac{1}{4} \frac{a^{4} U^{\prime \prime}(a)}{r^{2} U(r)} j_{\rho}(r) .
\end{aligned}
$$

In terms of these functions, the contributions to the stress tensor $T_{2}^{i j}$ become

$$
\begin{aligned}
& {\left[T_{2}^{i j}\right]^{s t}=} \\
& -\int_{\infty}^{a} d r\left\{\left(\rho^{2}-B^{2}\right)\left(-2 \frac{j_{\epsilon} j_{\epsilon}^{\prime}}{r}+2 \frac{j_{\epsilon} j_{\epsilon}}{r^{2}}+\frac{1}{2} j_{\epsilon}^{\prime} j_{\epsilon}^{\prime}\right)+2 U A_{\epsilon}^{2}+4 B^{2} \frac{A_{\epsilon} j_{\epsilon}}{r^{2}}\right\}\left[\partial_{i} \epsilon \partial_{j} \epsilon\right]^{s t} \\
& +\int_{\infty}^{a} d r\left\{\left(\rho^{2}-B^{2}\right)\left(-2 \frac{j_{\rho} j_{\rho}^{\prime}}{r}+2 \frac{j_{\rho} j_{\rho}}{r^{2}}+\frac{1}{2} j_{\rho}^{\prime} j_{\rho}^{\prime}\right)+2 U A_{\rho}^{2}+4 B^{2} \frac{A_{\rho} j_{\rho}}{r^{2}}+4 \frac{A_{\rho}}{r}\right\}\left[\partial_{i} \rho \partial_{j} \rho\right]^{s t} \\
& -\int_{\infty}^{a} d r\left\{\left(\rho^{2}-B^{2}\right)\left(-2 \frac{\left(j_{\epsilon} j_{\rho}^{\prime}+j_{\epsilon}^{\prime} j_{\rho}\right)}{r}+4 \frac{j_{\epsilon} j_{\rho}}{r^{2}}+j_{\epsilon}^{\prime} j_{\rho}^{\prime}\right)+4 U A_{\epsilon} A_{\rho}+4 \frac{A_{\epsilon}}{r}\right. \\
& -4 \rho B \int_{\infty}^{a} d r\left(\frac{j_{\epsilon} j_{\epsilon}^{\prime}}{r}-\frac{j_{\epsilon} j_{\epsilon}}{r^{2}}-\frac{1}{4} j_{\epsilon}^{\prime} j_{\epsilon}^{\prime}+\frac{A_{\epsilon} j_{\epsilon}}{r^{2}}\right)\left[\epsilon_{i k} \partial_{k} \epsilon \partial_{j} \epsilon\right]^{s t} \\
& +4 \rho B \int_{\infty}^{a} d r\left(\frac{j_{\rho} j_{\rho}^{\prime}}{r}-\frac{j_{\rho} j_{\rho}}{r^{2}}-\frac{1}{4} j_{\rho}^{\prime} j_{\rho}^{\prime}+\frac{A_{\rho} j_{\rho}}{r^{2}}\right)\left[\epsilon_{i k} \partial_{k} \rho \partial_{j} \rho\right]^{s t} \\
& +4 \rho B \int_{\infty}^{a} d r\left(\frac{\left(j_{\epsilon} j_{\rho}^{\prime}+j_{\epsilon}^{\prime} j_{\rho}\right)}{r}-2 \frac{j_{\epsilon} j_{\rho}}{r^{2}}-\frac{1}{2} j_{\epsilon}^{\prime} j_{\rho}^{\prime}+\frac{A_{\epsilon} j_{\rho}+A_{\rho} j_{\epsilon}}{r^{2}}\right)\left[\partial_{i} \epsilon \partial_{j} \rho\right]^{s t} .
\end{aligned}
$$

Adding these to the terms in (6.32), we can read off all of the second order nonlinear 
transport coefficients,

$$
\begin{aligned}
b_{3}^{\epsilon \epsilon}= & \frac{1}{4} \frac{\partial}{\partial \epsilon}\left(\frac{a(\epsilon, \rho)^{2}}{\rho^{2}+B^{2}}\right)-\int_{\infty}^{a} d r\left\{\left(\rho^{2}-B^{2}\right)\left(-2 \frac{j_{\epsilon} j_{\epsilon}^{\prime}}{r}+2 \frac{j_{\epsilon} j_{\epsilon}}{r^{2}}+\frac{1}{2} j_{\epsilon}^{\prime} j_{\epsilon}^{\prime}\right)+2 U A_{\epsilon}^{2}+4 B^{2} \frac{A_{\epsilon} j_{\epsilon}}{r^{2}}\right\} \\
b_{3}^{\epsilon \rho}= & {\left[\frac{1}{4} \frac{\partial}{\partial \rho}\left(\frac{a(\epsilon, \rho)^{2}}{\rho^{2}+B^{2}}\right)-\frac{1}{2} \frac{\partial}{\partial \epsilon}\left(\frac{a(\epsilon, \rho) \rho}{\rho^{2}+B^{2}}\right)\right] } \\
& +4 \rho B \int_{\infty}^{a} d r\left(\frac{\left(j_{\epsilon} j_{\rho}^{\prime}+j_{\epsilon}^{\prime} j_{\rho}\right)}{r}-2 \frac{j_{\epsilon} j_{\rho}}{r^{2}}-\frac{1}{2} j_{\epsilon}^{\prime} j_{\rho}^{\prime}+\frac{A_{\epsilon} j_{\rho}+A_{\rho} j_{\epsilon}}{r^{2}}\right) \\
b_{3}^{\rho \rho}= & -\frac{1}{2} \frac{\partial}{\partial \rho}\left(\frac{a(\epsilon, \rho) \rho}{\rho^{2}+B^{2}}\right) \\
& +\int_{\infty}^{a} d r\left\{\left(\rho^{2}-B^{2}\right)\left(-2 \frac{j_{\rho} j_{\rho}^{\prime}}{r}+2 \frac{j_{\rho} j_{\rho}}{r^{2}}+\frac{1}{2} j_{\rho}^{\prime} j_{\rho}^{\prime}\right)+2 U A_{\rho}^{2}+4 B^{2} \frac{A_{\rho} j_{\rho}}{r^{2}}+4 \frac{A_{\rho}}{r}\right\},
\end{aligned}
$$

and

$$
\begin{aligned}
b_{4}^{\epsilon \epsilon}= & \frac{1}{4 B} \frac{\partial}{\partial \epsilon}\left(\frac{a(\epsilon, \rho)^{2} \rho}{\rho^{2}+B^{2}}\right)-4 \rho B \int_{\infty}^{a} d r\left(\frac{j_{\epsilon} j_{\epsilon}^{\prime}}{r}-\frac{j_{\epsilon} j_{\epsilon}}{r^{2}}-\frac{1}{4} j_{\epsilon}^{\prime} j_{\epsilon}^{\prime}+\frac{A_{\epsilon} j_{\epsilon}}{r^{2}}\right) \\
b_{4}^{\epsilon \rho}= & \frac{1}{4 B} \frac{\partial}{\partial \rho}\left(\frac{a(\epsilon, \rho)^{2} \rho}{\rho^{2}+B^{2}}\right)+\frac{B}{2} \frac{\partial}{\partial \epsilon}\left(\frac{a(\epsilon, \rho)}{\rho^{2}+B^{2}}\right) \\
& \quad-\int_{\infty}^{a} d r\left\{\left(\rho^{2}-B^{2}\right)\left(-2 \frac{\left(j_{\epsilon} j_{\rho}^{\prime}+j_{\epsilon}^{\prime} j_{\rho}\right)}{r}+4 \frac{j_{\epsilon} j_{\rho}}{r^{2}}+j_{\epsilon}^{\prime} j_{\rho}^{\prime}\right)+4 U A_{\epsilon} A_{\rho}+4 \frac{A_{\epsilon}}{r}\right. \\
& \left.+4 B^{2} \frac{\left(A_{\epsilon} j_{\rho}+A_{\rho} j_{\epsilon}\right)}{r^{2}}\right\} \\
b_{4}^{\rho \rho}= & \frac{B}{2} \frac{\partial}{\partial \rho}\left(\frac{a(\epsilon, \rho)}{\rho^{2}+B^{2}}\right)+4 \rho B \int_{\infty}^{a} d r\left(\frac{j_{\rho} j_{\rho}^{\prime}}{r}-\frac{j_{\rho} j_{\rho}}{r^{2}}-\frac{1}{4} j_{\rho}^{\prime} j_{\rho}^{\prime}+\frac{A_{\rho} j_{\rho}}{r^{2}}\right) .
\end{aligned}
$$

The final step in the construction of our second order solution is to check that the remaining Einstein-Maxwell equations, $W_{M v}^{(2)}=Y^{(2) r}=0$, imply the equations of fluid dynamics (1.1) at order $\varepsilon^{2}$. This is indeed the case.

\section{Conclusion}

We have shown how the dynamics of a dyonic black brane can give rise to nonlinear magnetohydrodynamic equations of motion. We explicitly solved the Einstein-Maxwell equations to second order in the fluid dynamical derivative expansion, and from this we found expressions for the stress tensor and current to second and third order in derivatives respectively. These expressions yielded gravity predictions for the magnetohydrodynamic transport coefficients of charge and energy densities in a specific conformal fluid, coefficients 
which can in principle be compared with experimental results dealing with $2+1$ dimensional fluids near a quantum critical point. Our linear transport coefficients extend to higher order results already appearing in the literature, and a new set of nonlinear transport coefficients is now available as well. We found explicit closed form expressions for the second order linear transport coefficients, and integral expressions for the nonlinear ones.

Our work also helped to demonstrate the broad applicability of the mechanism [1] by which the equations of fluid dynamics emerge from gravity at long wavelengths. Compared to previous examples, one novelty of our setup was that it did not involve considering boosted black branes, due to the breaking of Lorentz invariance by the background magnetic field. Instead, we only needed to consider fluctuations of the energy and charge density.

Various generalizations and extensions of this work are possible, such as considering higher dimensional spacetimes, curved boundaries, etc. As with other examples, it may be interesting to consider in more detail particular solutions, and to see what can be learned about gravity from properties of the fluid dynamics, and vice versa.

\section{Acknowledgments:}

We thank Sean Hartnoll, Chris Herzog, Esko Keski-Vakkuri, and Markus Muller for helpful discussions and correspondence. Work of PK is supported in part by NSF grant PHY0456200 .

\section{Appendix A. Integrals}

Here we give the results for the integrals appearing in (6.7). First write $U$ in terms of its roots as $U=\frac{1}{r^{2}}\left(r-r_{1}\right)\left(r-r_{2}\right)\left(r-r_{3}\right)\left(r-r_{4}\right)$. We note that one of the roots is equal to $a$, while the other three can be obtained explicitly as the roots of a cubic equation.

We will use the identity

$$
\frac{1}{U^{2}}=\sum_{i} \frac{1}{\left[U^{\prime}\left(r_{i}\right)\right]^{2}} \frac{1}{\left(r-r_{i}\right)^{2}}-\sum_{i} \frac{U^{\prime \prime}\left(r_{i}\right)}{\left[U^{\prime}\left(r_{i}\right)\right]^{3}} \frac{1}{\left(r-r_{i}\right)}
$$

along with

$$
\begin{aligned}
& \sum_{i}\left(\frac{1}{\left[U^{\prime}\left(r_{i}\right)\right]^{2}} \frac{1}{r_{i}^{2}}+\frac{U^{\prime \prime}\left(r_{i}\right)}{\left[U^{\prime}\left(r_{i}\right)\right]^{3}} \frac{1}{r_{i}}\right)=0 \\
& \sum_{i}\left(2 \frac{1}{\left[U^{\prime}\left(r_{i}\right)\right]^{2}} \frac{1}{r_{i}^{3}}+\frac{U^{\prime \prime}\left(r_{i}\right)}{\left[U^{\prime}\left(r_{i}\right)\right]^{3}} \frac{1}{r_{i}^{2}}\right)=0 .
\end{aligned}
$$

The identities in (A.2) following from applying derivatives to (A.1), evaluated at $r=0$. We then find 


$$
\begin{aligned}
\int \frac{d r}{U^{2}} & =-\sum_{i} \frac{1}{\left[U^{\prime}\left(r_{i}\right)\right]^{2}} \frac{1}{\left(r-r_{i}\right)}-\sum_{i} \frac{U^{\prime \prime}\left(r_{i}\right)}{\left[U^{\prime}\left(r_{i}\right)\right]^{3}} \ln \left(r-r_{i}\right) \\
\int \frac{d r}{r U^{2}} & =-\sum_{i} \frac{1}{\left[U^{\prime}\left(r_{i}\right)\right]^{2}}\left(\frac{1}{r_{i}\left(r-r_{i}\right)}+\frac{\ln \left(r-r_{i}\right)}{r_{i}^{2}}\right)-\sum_{i} \frac{U^{\prime \prime}\left(r_{i}\right)}{\left[U^{\prime}\left(r_{i}\right)\right]^{3}} \frac{\ln \left(r-r_{i}\right)}{r_{i}} \\
\int \frac{d r}{r^{2} U^{2}} & =-\sum_{i} \frac{1}{\left[U^{\prime}\left(r_{i}\right)\right]^{2}}\left(\frac{1}{r_{i}^{2}\left(r-r_{i}\right)}+2 \frac{\ln \left(r-r_{i}\right)}{r_{i}^{3}}\right)-\sum_{i} \frac{U^{\prime \prime}\left(r_{i}\right)}{\left[U^{\prime}\left(r_{i}\right)\right]^{3}} \frac{\ln \left(r-r_{i}\right)}{r_{i}^{2}} .
\end{aligned}
$$




\section{References}

[1] S. Bhattacharyya, V. E. Hubeny, S. Minwalla and M. Rangamani, "Nonlinear Fluid Dynamics from Gravity," JHEP 0802, 045 (2008) [arXiv:0712.2456 [hep-th]].

[2] S. Bhattacharyya et al., "Local Fluid Dynamical Entropy from Gravity," JHEP 0806, 055 (2008) arXiv:0803.2526 [hep-th]].

[3] M. Van Raamsdonk, "Black Hole Dynamics From Atmospheric Science," JHEP 0805, 106 (2008) [arXiv:0802.3224 [hep-th]].

[4] M. Haack and A. Yarom, "Nonlinear viscous hydrodynamics in various dimensions using AdS/CFT," JHEP 0810, 063 (2008) arXiv:0806.4602 [hep-th]].

[5] S. Bhattacharyya, R. Loganayagam, I. Mandal, S. Minwalla and A. Sharma, "Conformal Nonlinear Fluid Dynamics from Gravity in Arbitrary Dimensions," arXiv:0809.4272 [hep-th].

[6] J. Erdmenger, M. Haack, M. Kaminski and A. Yarom, "Fluid dynamics of R-charged black holes," arXiv:0809.2488 [hep-th].

[7] N. Banerjee, J. Bhattacharya, S. Bhattacharyya, S. Dutta, R. Loganayagam and P. Surowka, "Hydrodynamics from charged black branes," arXiv:0809.2596 [hep-th].

[8] M. Haack and A. Yarom, "Universality of second order transport coefficients from the gauge-string duality," arXiv:0811.1794 [hep-th].

[9] S. Bhattacharyya, R. Loganayagam, S. Minwalla, S. Nampuri, S. P. Trivedi and S. R. Wadia, "Forced Fluid Dynamics from Gravity," arXiv:0806.0006 [hep-th].

[10] D. T. Son and A. O. Starinets, "Viscosity, Black Holes, and Quantum Field Theory," Ann. Rev. Nucl. Part. Sci. 57, 95 (2007) [arXiv:0704.0240 [hep-th]].

[11] C. P. Herzog, P. Kovtun, S. Sachdev and D. T. Son, "Quantum critical transport, duality, and M-theory," Phys. Rev. D 75, 085020 (2007) arXiv:hep-th/0701036].

[12] S. A. Hartnoll and P. Kovtun, "Hall conductivity from dyonic black holes," Phys. Rev. D 76, 066001 (2007) arXiv:0704.1160 [hep-th]].

[13] S. A. Hartnoll, P. K. Kovtun, M. Muller and S. Sachdev, "Theory of the Nernst effect near quantum phase transitions in condensed matter, and in dyonic black holes," Phys. Rev. B 76, 144502 (2007) [arXiv:0706.3215 [cond-mat.str-el]].

[14] S. A. Hartnoll and C. P. Herzog, "Ohm's Law at strong coupling: S duality and the cyclotron resonance," Phys. Rev. D 76, 106012 (2007) arXiv:0706.3228 [hep-th]].

[15] E. I. Buchbinder, A. Buchel and S. E. Vazquez, "Sound Waves in $(2+1)$ Dimensional Holographic Magnetic Fluids," arXiv:0810.4094 [hep-th].

[16] need to supply reference BuchbinderNF.

[17] V. Balasubramanian and P. Kraus, "A stress tensor for anti-de Sitter gravity," Commun. Math. Phys. 208, 413 (1999) arXiv:hep-th/9902121.

[18] M. Henningson and K. Skenderis, "The holographic Weyl anomaly," JHEP 9807, 023 (1998) arXiv:hep-th/9806087. 
[19] G. T. Horowitz and V. E. Hubeny, "Quasinormal modes of AdS black holes and the approach to thermal equilibrium," Phys. Rev. D 62, 024027 (2000) arXiv:hepth/9909056].

[20] E. Witten, "SL $(2, \mathrm{Z})$ action on three-dimensional conformal field theories with Abelian symmetry," arXiv:hep-th/0307041. 